\title{
General boundary quantum field theory: Timelike hypersurfaces in Klein-Gordon theory
}

\author{
Robert Oeckl* \\ Instituto de Matemáticas, UNAM Campus Morelia, \\ C.P. 58190, Morelia, Michoacán, Mexico
}

15 September 2005

9 March 2006 (v2)

\begin{abstract}
We show that the real massive Klein-Gordon theory admits a description in terms of states on various timelike hypersurfaces and amplitudes associated to regions bounded by them. This realizes crucial elements of the general boundary framework for quantum field theory. The hypersurfaces considered are hyperplanes on the one hand and timelike hypercylinders on the other hand. The latter lead to the first explicit examples of amplitudes associated with finite regions of space, and admit no standard description in terms of "initial" and "final" states. We demonstrate a generalized probability interpretation in this example, going beyond the applicability of standard quantum mechanics.
\end{abstract}

*email: robert@matmor.unam.mx 


\section{Contents}

1 Introduction 3

2 Generalized Schrödinger-Fevnman approach 4

2.1 States and amplitudes . . . . . . . . . . . . . . . 4

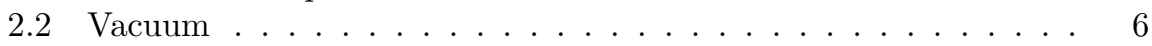

\begin{tabular}{lll}
\hline 3 & Spacelike hvperplanes & 7
\end{tabular}

3.1 Propagator . . . . . . . . . . . . . . . . . . 7

3.2 Vacuum . . . . . . . . . . . . . . . . 8

3.3 Particle states . . . . . . . . . . . . . . . . . . . . 9

$\begin{array}{lll}4 \text { General hyperplanes } & 10\end{array}$

4.1 Timelike propagaton . . . . . . . . . . . . . . . . . . 11

4.2 Timelike vacuum . . . . . . . . . . . . . . . . . . . . 11

4.3 Timelike particle states . . . . . . . . . . . . . . . . . 12

4.4 General vacuum $\ldots \ldots \ldots \ldots$

$\begin{array}{lll}5 \text { The hvpercvlinder } & 14\end{array}$

5.1 Coordinates classical solutions etc. . . . . . . . . . . . . . . . 14

5.2 Propagators . . . . . . . . . . . . . . . . . . . . 15

5.3 Vacuum . . . . . . . . . . . . . . . . . . . . . . 17

5.4 Particle states . . . . . . . . . . . . . . . . . . . . . . 19

$\begin{array}{lll}6 & \text { Interpretation } & 20\end{array}$

6.1 Particles on timelike hypersurfaces . . . . . . . . . . . . 20

6.2 Probabilitv interpretation . . . . . . . . . . . . . . . 21

$\begin{array}{lll}7 \text { Conclusions and Outlook } & 24\end{array}$ 


\section{Introduction}

State spaces in quantum field theory are normally associated with spacelike hypersurfaces. What is more, in flat spacetime one usually only considers one state space, identifying all of them through time-translation symmetry. The restriction to spacelike hypersurfaces has various reasons, among them the necessity to conserve probabilities in the standard formulation of quantum mechanics.

In contrast, we contend that this restriction is artificial. Indeed, we have shown in [1] that considering states on certain timelike hypersurfaces seems to make perfect sense. The example considered was the real massive Klein-Gordon theory and the hypersurfaces discussed were hyperplanes. In particular, we found a consistent vacuum state on arbitrary hyperplanes and elucidated the nature of particles states on timelike hyperplanes.

An underlying framework into which these results may be fitted is the so called general boundary formulation of quantum mechanics, also called general boundary quantum field theory. The foundations of this framework are laid out in the companion paper [2]. However, the present paper should be readable independently.

The basic idea of this approach, going back to 3,4 is that transition amplitudes make sense not only between instances of time, but may be associated to regions of spacetime which are not necessarily defined by a time interval. Furthermore, the relevant state spaces are associated to the boundary hypersurfaces of these regions.

In the present article we continue the development of Klein-Gordon theory in this framework. The key new example of hypersurface we consider is an infinite timelike hypercylinder. More precisely, consider a sphere in space and extend it over all of time. This is what we call the hypercylinder. The spacetime regions of interest in this context are the solid hypercylinder as well as a solid hypercylinder with another one cut out.

Using the Schrödinger representation combined with the Feynman path integral (explained in Section 2) we work out state spaces of wave functions, field propagators, vacuum states and particle states. We show that all these structures are consistent in the sense of general boundary quantum field theory. In doing so, we start by reviewing spacelike hyperplanes (Section 3) to clarify the approach, then move to recall and elaborate on the general hyperplane case (Section 4). The main technical results are obtained in the treatment of the hypercylinder case (Section 5), with the novel situation of an amplitude associated to a region of spacetime with connected boundary. This case requires a genuinely new interpretation and the general boundary formulation shows its full force here.

After the technical part we move to a discussion of the interpretation (Section (6). This consists firstly of a discussion of the meaning of particle states on timelike hypersurfaces. In particular, we elaborate on the fact that particles within a state acquire the property of being incoming or outgoing individually. Secondly, we apply the probability interpretation proposed in [2] to the case of the solid hypercylinder. Due to the connectedness of the boundary an amplitude 
is to be evaluated on a single state. While this is out of the range of applicability of standard quantum mechanics, the proposed interpretation yields a physically fully satisfactory answer. We close with a few remarks (Section $\mathbf{7}$ ).

\section{Generalized Schrödinger-Feynman approach}

Our aim is to show how the Klein-Gordon theory admits a generalized description in terms of a general boundary quantum field theory. More precisely, we wish to show how it admits states on hypersurfaces that are not necessarily spacelike and amplitudes associated to spacetime regions which are not necessarily time intervals. It turns out that to this end the Schrödinger representation (where states are wave functions) combined with the Feynman path integral [5] are particularly suitable [4. Since the former is not usually employed in quantum field theory, we refer to the review [6].

We start by introducing the basic structures and their heuristic definition. This includes state spaces, inner products, propagators etc. Most of these exist already in the standard formalism, but will be defined here in a generalized way. Furthermore, consistency conditions will be formulated that these structures must satisfy. These conditions may be derived directly from the axiomatic framework presented in the companion paper [2, see also Section 7 of that paper. Again, these generalize consistency conditions of the standard quantum mechanical framework, including unitarity, composability of time evolutions etc. That this generalization really makes sense is the subject of the remaining part of this article.

\subsection{States and amplitudes}

The basic spacetime objects we will need are hypersurfaces $\Sigma$ and regions (4submanifolds) $M$ in Minkowski space. The former generalize spacelike hypersurfaces and the latter generalize regions of time-evolution between them.

On a given hypersurface $\Sigma$ we consider the space of field configurations, which we denote by $K_{\Sigma}$. Since we are dealing with a theory of one real scalar field this is basically the space of real-valued functions on $\Sigma .^{1}$ The Schrödinger representation prescribes now that states are complex-valued wave functions on this configuration space. Thus, we associate with $\Sigma$ its space of states (wave functions) which we denote $\mathcal{H}_{\Sigma}$. This state space carries an inner product, defined through an integral over field configurations,

$$
\left\langle\psi, \psi^{\prime}\right\rangle_{\Sigma}:=\int_{K_{\Sigma}} \mathcal{D} \varphi \overline{\psi(\varphi)} \psi^{\prime}(\varphi)
$$

Note the following important property of state spaces. Take a hypersurface $\Sigma$ which is the disjoint union of two hypersurfaces $\Sigma_{1} \cup \Sigma_{2}$. The field configurations on $\Sigma$ are then obviously pairs of field configurations on $\Sigma_{1}$ and on $\Sigma_{2}$, i.e.,

\footnotetext{
${ }^{1}$ We will later see that this naive definition is not always correct. However, it will suffice here for the intuitive picture.
} 
$K_{\Sigma}=K_{\Sigma_{1}} \times K_{\Sigma_{2}}$. Thus, wave functions on $\Sigma$ can be expanded into products of wave functions on $\Sigma_{1}$ and $\Sigma_{2}$, i.e., $\mathcal{H}_{\Sigma}=\mathcal{H}_{\Sigma_{1}} \otimes \mathcal{H}_{\Sigma_{2}}$.

We will make use of another structure on hypersurfaces: orientation. From now on we will think of each hypersurfaces as oriented, i.e., each hypersurface has a chosen "side". For a given oriented hypersurface $\Sigma$, we denote its oppositely oriented version by $\bar{\Sigma}$, i.e., using an over-bar. Although the associated state spaces $\mathcal{H}_{\Sigma}$ and $\mathcal{H}_{\bar{\Sigma}}$ are canonically identical (indeed, so far we have not distinguished between them), their physical meaning must be distinguished in the following. However, there is a map that tells us which state on $\bar{\Sigma}$ corresponds physically to a given state on $\Sigma$. This map, denoted by $\iota_{\Sigma}: \mathcal{H}_{\Sigma} \rightarrow \mathcal{H}_{\bar{\Sigma}}$ is given by the complex conjugation of wave functions,

$$
\left(\iota_{\Sigma}(\psi)\right)(\varphi):=\overline{\psi(\varphi)} \quad \forall \psi \in \mathcal{H}_{\Sigma}, \varphi \in K_{\Sigma} .
$$

Let $M$ be a region with boundary $\Sigma$. The hypersurface $\Sigma$ is oriented by choosing the "outside" of $M$. We define the amplitude associated with $M$ as the map $\rho_{M}: \mathcal{H}_{\Sigma} \rightarrow \mathbb{C}$, associating with each wave function a complex number as follows,

$$
\begin{aligned}
\rho_{M}(\psi) & :=\int_{K_{\Sigma}} \mathcal{D} \varphi \psi(\varphi) Z_{M}(\varphi) \quad \forall \psi \in \mathcal{H}_{\Sigma}, \\
Z_{M}(\varphi) & :=\int_{K_{M},\left.\phi\right|_{\Sigma}=\varphi} \mathcal{D} \phi e^{\mathrm{i} S_{M}(\phi)} \quad \forall \varphi \in K_{\Sigma} .
\end{aligned}
$$

The second integral is the Feynman path integral over "all field configurations" $\phi \in K_{M}$ in the region $M$ that reduce to $\varphi$ on the boundary $\Sigma . S_{M}$ is the action integral over the region $M$. The quantity $Z_{M}(\varphi)$ is called the field propagator. It formally looks like a wave function, but might not be normalizable with respect to the inner product (10).

Consider a region $M$ with boundary $\Sigma$ consisting of the disjoint union of two components $\Sigma_{1}$ and $\Sigma_{2}$. Then, the amplitude $\rho_{M}: \mathcal{H}_{\Sigma_{1}} \otimes \mathcal{H}_{\Sigma_{2}} \rightarrow \mathbb{C}$ induces a $\operatorname{map} \tilde{\rho}_{M}: \mathcal{H}_{\Sigma_{1}} \rightarrow \mathcal{H}_{\bar{\Sigma}_{2}}$ via

$$
\left(\tilde{\rho}_{M}(\psi)\right)\left(\varphi^{\prime}\right)=\int_{K_{\Sigma_{1}}} \mathcal{D} \varphi \psi(\varphi) Z_{M}\left(\varphi, \varphi^{\prime}\right) \quad \forall \psi \in \mathcal{H}_{\Sigma_{1}}, \varphi^{\prime} \in K_{\bar{\Sigma}_{2}} .
$$

Note that the orientation reversal on $\Sigma_{2}$ comes from the fact that the Hilbert space $\mathcal{H}_{\Sigma_{2}}$ must be dualized when moved from the domain of $\rho_{M}$ to the image. But the inner product (1) together with the conjugation (2) make $\mathcal{H}_{\bar{\Sigma}_{2}}$ precisely into the dual space of $\mathcal{H}_{\Sigma_{2}}$. The physical meaning of $\tilde{\rho}_{M}$ is that (in suitable circumstances) we may think of it as describing the evolution (not necessarily in time) of the system from $\Sigma_{1}$ to $\bar{\Sigma}_{2}$.

In particular, suppose we have two spacelike hypersurfaces $\Sigma_{1}$ and $\bar{\Sigma}_{2}$ and call the intermediate region $M$. Then, $\tilde{\rho}_{M}(\psi)$ is the time-evolved wave function on $\bar{\Sigma}_{2}$ for the initial wave function $\psi$ on $\Sigma_{1}$. Note here that $\Sigma_{1}$ and $\bar{\Sigma}_{2}$ have the same orientation with respect to the time direction, namely with their "past" side selected. Indeed, the reason why the orientation of hypersurfaces does not 
appear explicitly in the standard formulation is that all spacelike hypersurfaces can be coherently oriented in the same way, using the time direction.

We now formulate what a unitarity evolution (i.e., an evolution preserving the inner product) means. One can check that for $\tilde{\rho}_{M}$ to be unitary the propagator must satisfy the following formal condition,

$$
\int_{K_{\Sigma_{2}}} \mathcal{D} \varphi_{2} \overline{Z_{M}\left(\varphi_{1}, \varphi_{2}\right)} Z_{M}\left(\varphi_{1}^{\prime}, \varphi_{2}\right)=\delta\left(\varphi_{1}, \varphi_{1}^{\prime}\right) \quad \forall \varphi_{1}, \varphi_{1}^{\prime} \in K_{\Sigma_{1}}
$$

Finally, consider a region $M_{1}$ with boundary consisting of disconnected components $\Sigma_{1}$ and $\Sigma$ and a region $M_{2}$ with boundary consisting of disconnected components $\bar{\Sigma}$ and $\Sigma_{2}$ such that $M_{1}$ and $M_{2}$ may be glued along $\Sigma$ to form a new region $M$. We then want that the evolution associated with $M$ is the composition of the evolutions associated with $M_{1}$ and $M_{2}$. That is, $\tilde{\rho}_{M_{1} \cup M_{2}}=\tilde{\rho}_{M_{2}} \circ \tilde{\rho}_{M_{1}}$. In terms of propagators this means,

$$
\int_{K_{\bar{\Sigma}}} \mathcal{D} \varphi Z_{M_{1}}\left(\varphi_{1}, \varphi\right) Z_{M_{2}}\left(\varphi, \varphi_{2}\right)=Z_{M_{1} \cup M_{2}}\left(\varphi_{1}, \varphi_{2}\right) \quad \forall \varphi_{1} \in K_{\Sigma_{1}}, \varphi_{2} \in K_{\bar{\Sigma}_{2}} .
$$

If we write the propagator in terms of the path integral (4) the validity of (7) becomes obvious. ${ }^{2}$

\subsection{Vacuum}

We postulate that there is a distinguished vacuum wave function on each hypersurface $\Sigma$, denoted $\psi_{\Sigma, 0}$. We require the vacuum to satisfy certain properties (the vacuum axioms of [2]). This is, firstly, the compatibility with conjugation. This means that the vacuum wave function on a hypersurface is complex conjugate to the vacuum on the hypersurface with the opposite orientation. Formally,

$$
\psi_{\bar{\Sigma}, 0}(\varphi)=\left(\iota_{\Sigma}\left(\psi_{\Sigma, 0}\right)\right)(\varphi)=\overline{\psi_{\Sigma, 0}(\varphi)} \quad \forall \varphi \in K_{\Sigma}
$$

Another property of the vacuum state we expect is that for a hypersurface $\Sigma$ consisting of disjoint hypersurfaces $\Sigma_{1}$ and $\Sigma_{2}$ the vacuum wave function should be the product of the individual vacuum wave functions, i.e.,

$$
\psi_{\Sigma, 0}\left(\varphi_{1}, \varphi_{2}\right)=\psi_{\Sigma_{1}, 0}\left(\varphi_{1}\right) \psi_{\Sigma_{2}, 0}\left(\varphi_{2}\right) \quad \forall \varphi_{1} \in K_{\Sigma_{1}}, \varphi_{2} \in K_{\Sigma_{2}}
$$

We also want the vacuum states to be normalized, i.e.,

$$
\int_{K_{\Sigma}} \mathcal{D} \varphi\left|\psi_{\Sigma, 0}(\varphi)\right|^{2}=1
$$

Finally, the amplitude of the vacuum state should be unity. Suppose $M$ is a region with boundary $\Sigma$, then

$$
\rho_{M}\left(\psi_{\Sigma, 0}\right)=\int_{K_{\Sigma}} \mathcal{D} \varphi \psi_{\Sigma, 0}(\varphi) Z_{M}(\varphi)=1
$$

\footnotetext{
${ }^{2}$ However, it becomes much less obvious when going beyond the naive picture presented here. We will see this in the following.
} 
As can be shown 2] these properties imply that the vacuum is preserved under evolution in the sense described above. That is, evolving from a hypersurface $\Sigma_{1}$ to a hypersurface $\bar{\Sigma}_{2}$ via a region $M$ with evolution map $\tilde{\rho}_{M}: \mathcal{H}_{\Sigma_{1}} \rightarrow \mathcal{H}_{\bar{\Sigma}_{2}}$, the vacuum satisfies

$$
\psi_{\bar{\Sigma}_{2}, 0}\left(\varphi_{2}\right)=\left(\tilde{\rho}_{M}\left(\psi_{\Sigma_{1}, 0}\right)\right)\left(\varphi_{2}\right)=\int_{K_{\Sigma_{1}}} \mathcal{D} \varphi_{1} \psi_{\Sigma_{1}, 0}\left(\varphi_{1}\right) Z_{M}\left(\varphi_{1}, \varphi_{2}\right) \quad \forall \varphi_{2} \in K_{\Sigma_{2}}
$$

In the context of parallel equal-time hyperplanes this is the standard notion of time-evolution invariance of the vacuum.

\section{$3 \quad$ Spacelike hyperplanes}

In this section we review standard elements of the Schrödinger representation of the Klein-Gordon theory. This will make the later generalization and its meaning more transparent. We follow here a presentation close to [1]. Hypersurfaces are here equal-time hyperplanes and regions are time intervals extended over all of space. We use time translation symmetry to identify the spaces of wave functions associated to all (past oriented) equal-time hyperplanes. (See 2] for a more detailed discussion of this identification.)

We start by recalling elementary features of the classical Klein-Gordon theory of a real scalar field $\phi$ with mass $m$ in Minkowski space. The equations of motion are given by the Klein-Gordon equation, $\left(\square+m^{2}\right) \phi=0$, with

$\square:=\partial_{0}^{2}-\sum_{i \geq 1} \partial_{i}^{2}$. The action on a region $M$ of Minkowski space is given by

$$
S_{M}(\phi)=\frac{1}{2} \int_{M} \mathrm{~d}^{4} x\left(\left(\partial_{0} \phi\right)\left(\partial_{0} \phi\right)-\sum_{i \geq 1}\left(\partial_{i} \phi\right)\left(\partial_{i} \phi\right)-m^{2} \phi^{2}\right) .
$$

We may rewrite this as follows,

$$
S_{M}(\phi)=-\frac{1}{2} \int_{M} \mathrm{~d}^{4} x \phi\left(\square+m^{2}\right) \phi+\frac{1}{2} \int_{\partial M} \mathrm{~d}^{3} x \phi\left(n_{0} \partial_{0}-\sum_{i \geq 1} n_{i} \partial_{i}\right) \phi .
$$

Here $n_{i}$ is the local euclidean normal vector to the boundary $\partial M$ of $M$ pointing outwards. Note that by the equations of motion, the action applied to a solution reduces to the boundary term.

\subsection{Propagator}

Consider two instants in time $t$ and $t^{\prime}$. The time interval $\left[t, t^{\prime}\right]$ defines a region in Minkowski space in the obvious way. Its boundary has two connected components associated with the two instants of time. Using the variational principle that determines the equations of motions together with the fact that the action is quadratic we can evaluate the field propagator (44) for this region. Choosing 
a classical solution $\phi_{\mathrm{cl}}$ matching the boundary data at $t$ and $t^{\prime}$ and shifting the integration variable yields

$$
\begin{aligned}
Z_{\left[t, t^{\prime}\right]}\left(\varphi, \varphi^{\prime}\right) & =\int_{\left.\phi\right|_{t}=\varphi,\left.\phi\right|_{t^{\prime}}=\varphi^{\prime}} \mathcal{D} \phi e^{\mathrm{i} S_{\left[t, t^{\prime}\right]}(\phi)} \\
& =\int_{\left.\phi\right|_{t}=0,\left.\phi\right|_{t^{\prime}}=0} \mathcal{D} \phi e^{\mathrm{i} S_{\left[t, t^{\prime}\right]}\left(\phi_{\mathrm{cl}}+\phi\right)} \\
& =N_{\left[t, t^{\prime}\right]} e^{\mathrm{i} S_{\left[t, t^{\prime}\right]}\left(\phi_{\mathrm{cl}}\right)},
\end{aligned}
$$

where $\Delta:=t^{\prime}-t$. The normalization factor is formally given by

$$
N_{\left[t, t^{\prime}\right]}=\int_{\left.\phi\right|_{t}=0,\left.\phi\right|_{t^{\prime}}=0} \mathcal{D} \phi e^{\mathrm{i} S_{\left[t, t^{\prime}\right]}(\phi)} .
$$

Using (13) the evaluation of the action on a classical solution reduces to a boundary integral. In the present case this is

$$
S_{\left[t, t^{\prime}\right]}\left(\phi_{\mathrm{cl}}\right)=\frac{1}{2} \int_{t^{\prime}} \mathrm{d}^{3} x \phi_{\mathrm{cl}}\left(t^{\prime}, x\right) \partial_{0} \phi_{\mathrm{cl}}\left(t^{\prime}, x\right)-\frac{1}{2} \int_{t} \mathrm{~d}^{3} x \phi_{\mathrm{cl}}(t, x) \partial_{0} \phi_{\mathrm{cl}}(t, x) .
$$

We split the classical solution into positive and negative energy components

$$
\phi_{\mathrm{cl}}(t, x)=e^{-\mathrm{i} \omega t} \varphi^{+}(x)+e^{\mathrm{i} \omega t} \varphi^{-}(x),
$$

where $\omega$ is the operator

$$
\omega:=\sqrt{-\sum_{i \geq 1} \partial_{i}^{2}+m^{2}} .
$$

Inserting this decomposition into (15) and inverting the formal linear transformation

$$
\left(\begin{array}{c}
\varphi \\
\varphi^{\prime}
\end{array}\right)=\left(\begin{array}{ll}
e^{-\mathrm{i} \omega t} & e^{\mathrm{i} \omega t} \\
e^{-\mathrm{i} \omega t^{\prime}} & e^{\mathrm{i} \omega t^{\prime}}
\end{array}\right)\left(\begin{array}{l}
\varphi^{+} \\
\varphi^{-}
\end{array}\right)
$$

yields the field propagator (see [4),

$$
Z_{\left[t, t^{\prime}\right]}\left(\varphi, \varphi^{\prime}\right)=N_{\left[t, t^{\prime}\right]} \exp \left(-\frac{1}{2} \int \mathrm{d}^{3} x\left(\begin{array}{ll}
\varphi & \varphi^{\prime}
\end{array}\right) W_{\left[t, t^{\prime}\right]}\left(\begin{array}{c}
\varphi \\
\varphi^{\prime}
\end{array}\right)\right) .
$$

The operator-valued matrix $W_{\left[t, t^{\prime}\right]}$ is given by

$$
W_{\left[t, t^{\prime}\right]}=\frac{-\mathrm{i} \omega}{\sin \omega \Delta}\left(\begin{array}{cc}
\cos \omega \Delta & -1 \\
-1 & \cos \omega \Delta
\end{array}\right)
$$

\subsection{Vacuum}

To obtain the vacuum wave function consider the Gaussian ansatz

$$
\psi_{0}(\varphi)=C \exp \left(-\frac{1}{2} \int \mathrm{d}^{3} x \varphi(x)(A \varphi)(x)\right)
$$


for an unknown operator $A$ and a normalization constant $C$. Imposing the time-evolution invariance (12) yields the equation $A^{2}=\omega^{2}$. We choose $A=\omega$, following the standard conventions. At the same time, this fixes the normalization factor $N_{\left[t, t^{\prime}\right]}$ to be formally

$$
N_{\left[t, t^{\prime}\right]}^{-1}=\int \mathcal{D} \varphi \exp \left(-\frac{1}{2} \int \mathrm{d}^{3} x \varphi(x) \frac{\omega \exp (\mathrm{i} \omega \Delta)}{\mathrm{i} \sin (\omega \Delta)} \varphi(x)\right) .
$$

One can check that with this normalization, the propagator satisfies both the unitarity condition (6) and the composition property (7).

The normalization condition (10) for the vacuum fixes the factor $C$ (up to a phase),

$$
|C|^{-2}=\int \mathcal{D} \varphi \exp \left(-\frac{1}{2} \int \mathrm{d}^{3} x \varphi(x)(2 \omega \varphi)(x)\right) .
$$

We choose $C$ to be real. We will come back to the reason for this later. Note that this implies that the vacuum wave function is real and thus (implementing (8) ) the same for both orientations of an equal-time hyperplane.

The property (9) is now a definition that only comes into force if we consider unions of different equal-time hyperplanes. The unit amplitude property (11) follows from the other properties already implemented.

\subsection{Particle states}

The particle states can be found by use of suitable creation and annihilation operators (see [6]) or through an expansion of the propagator in terms of eigenstates. We will not enter into the details here.

Using the Fourier transform,

$$
\check{\varphi}(p)=2 E \int \mathrm{d}^{3} x e^{\mathrm{i} p x} \varphi(x), \quad \varphi(x)=\int \frac{\mathrm{d}^{3} p}{(2 \pi)^{3} 2 E} e^{-\mathrm{i} p x} \check{\varphi}(p)
$$

the one-particle wave function of momentum $p$ is given by

$$
\psi_{p}(\varphi)=\check{\varphi}(p) \psi_{0}(\varphi)
$$

These wave functions have a distributional normalization, given by

$$
\left\langle\psi_{p}, \psi_{p^{\prime}}\right\rangle=(2 \pi)^{3} 2 E \delta^{3}\left(p-p^{\prime}\right) .
$$

The two-particle state with momenta $p$ and $p^{\prime}$ is given by the wave function

$$
\psi_{p, p^{\prime}}(\varphi)=\left(\check{\varphi}(p) \check{\varphi}\left(p^{\prime}\right)-(2 \pi)^{3} 2 E \delta^{3}\left(p+p^{\prime}\right)\right) \psi_{0}(\varphi) .
$$

The $n$-particle wave function is a polynomial of degree $n$ in Fourier transforms times the vacuum wave function. To obtain it explicitly for given momenta $p_{1}, \ldots, p_{n}$, one may project the wave function

$$
\check{\varphi}\left(p_{1}\right) \cdots \check{\varphi}\left(p_{n}\right) \psi_{0}(\varphi)
$$


to the orthogonal complement of the spaces of states with less than $n$ particles.

In contrast to the vacuum, particle wave functions are generically not real. Thus, (2) prescribes that a given particle wave function change explicitly to its complex conjugate when considered on an equal-time hyperplane with opposite orientation. Since we have taken "past-orientation" as standard, we denote a wave function $\psi$, when specifying the same state but with "future-orientation" by $\bar{\psi}$.

The transition amplitude from an $n$-particle state $\psi_{p_{1}, \ldots, p_{n}}$ at time $t_{1}$ to to an $m$-particle state $\psi_{q_{1}, \ldots, q_{m}}$ is given by the amplitude function (3) and evaluates to

$$
\begin{aligned}
& \rho_{\left[t, t^{\prime}\right]}\left(\psi_{p_{1}, \ldots, p_{n}} \otimes \bar{\psi}_{q_{1}, \ldots, q_{m}}\right) \\
& =\int_{K_{t} \times K_{t^{\prime}}} \mathcal{D} \varphi \mathcal{D} \varphi^{\prime} \psi_{p_{1}, \ldots, p_{n}}(\varphi) \overline{\psi_{q_{1}, \ldots, q_{m}}\left(\varphi^{\prime}\right)} Z_{\left[t, t^{\prime}\right]}\left(\varphi, \varphi^{\prime}\right) \\
& \quad=\delta_{n, m} e^{-\mathrm{i} \sum_{i=1}^{n} \Delta E_{i}} \sum_{\sigma \in S_{n}} \prod_{i=1}^{n}(2 \pi)^{3} 2 E_{i} \delta^{3}\left(p_{i}-q_{\sigma(i)}\right) .
\end{aligned}
$$

The sum runs over all permutations $\sigma$ of $n$ elements.

Using (5) the notation for the amplitude may be brought into the more conventional form

$$
\rho_{\left[t, t^{\prime}\right]}\left(\psi_{p_{1}, \ldots, p_{n}} \otimes \bar{\psi}_{q_{1}, \ldots, q_{m}}\right)=\left\langle\psi_{q_{1}, \ldots, q_{m}}, \tilde{\rho}_{\left[t, t^{\prime}\right]}\left(\psi_{p_{1}, \ldots, p_{n}}\right)\right\rangle
$$

which recovers the usual bra-ket notation.

\section{General hyperplanes}

We now generalize from equal-time hyperplanes to arbitrary hyperplanes in Minkowski space 1]. We start with a particular timelike hyperplane.

Consider the hyperplane aligned with the time axis and spanned by coordinate directions $\left(t, x_{2}, x_{3}\right)$. We will denote the coordinates $x_{2}, x_{3}$ collectively by $\tilde{x}$. A crucial difference to the spacelike case arises as follows. When considering field configurations in the sense of Section 2 we have to keep in mind that a field configuration together with the conjugate momentum should correspond to a classical solution. If we consider configuration and momentum on a spacelike hyperplane, then clearly, a configuration can be essentially any real function on the hyperplane. However, on a timelike hyperplane not every real function extends to a classical solution. It is easy to see that the space of configurations that do extend is the subspace of the space of "all" configurations on which the square of the operator

$$
\kappa_{1}:=\sqrt{-\partial_{0}^{2}+\sum_{i \geq 2} \partial_{i}^{2}-m^{2}}
$$

has non-negative eigenvalues. (Thus, $\kappa_{1}$ itself is well defined on this space.) We call this the physical configuration space. 


\subsection{Timelike propagator}

The first object of interest in this context is the propagator, say for the spacetime region defined by the interval $\left[x_{1}, x_{1}^{\prime}\right]$. It was shown in [1] that it can be calculated along lines entirely analogous to the equal-time hyperplane case. Namely, the path integral can be evaluated with the help of a classical solution matching the boundary data. This involves using the boundary form (13) of the action evaluated on the classical solution. This time, the step analogous to the decomposition (16) is the decomposition in terms of solutions with positive versus negative momentum in the $x_{1}$-direction,

$$
\phi(t, x)=e^{\mathrm{i} \kappa_{1} x_{1}} \varphi^{+}(t, \tilde{x})+e^{-\mathrm{i} \kappa_{1} x_{1}} \varphi^{-}(t, \tilde{x}) .
$$

This leads to the result,

$$
Z_{\left[x_{1}, x_{1}^{\prime}\right]}\left(\varphi, \varphi^{\prime}\right)=N_{\left[x_{1}, x_{1}^{\prime}\right]} \exp \left(-\frac{1}{2} \int \mathrm{d} t \mathrm{~d}^{2} \tilde{x}\left(\begin{array}{ll}
\varphi & \varphi^{\prime}
\end{array}\right) W_{\left[x_{1}, x_{1}^{\prime}\right]}\left(\begin{array}{c}
\varphi \\
\varphi^{\prime}
\end{array}\right)\right),
$$

with $\Delta:=\left|x_{1}^{\prime}-x_{1}\right|$. The operator-valued matrix $W_{\left[x_{1}, x_{1}^{\prime}\right]}$ is given by

$$
W_{\left[x_{1}, x_{1}^{\prime}\right]}=\frac{\mathrm{i} \kappa_{1}}{\sin \kappa_{1} \Delta}\left(\begin{array}{cc}
\cos \kappa_{1} \Delta & -1 \\
-1 & \cos \kappa_{1} \Delta
\end{array}\right) .
$$

\subsection{Timelike vacuum}

To determine the vacuum state we make the ansatz analogous to (18), i.e., using a Gaussian bilinear form with undetermined operator $A$. Explicit computation yields $A^{2}=\kappa_{1}^{2}$. We choose $A=\kappa_{1}$, justifying this choice later. Note that this also fixes the normalization factor $N_{\left[x_{1}, x_{1}^{\prime}\right]}$ appearing in the propagator to

$$
N_{\left[x_{1}, x_{1}^{\prime}\right]}^{-1}=\int \mathcal{D} \varphi \exp \left(-\frac{1}{2} \int \mathrm{d} t \mathrm{~d}^{2} \tilde{x} \varphi \frac{\mathrm{i} \kappa_{1} \exp \left(-\mathrm{i} \kappa_{1} \Delta\right)}{\sin \kappa_{1} \Delta} \varphi\right) .
$$

One can check now that the unitarity condition (6) as well as the composition property (77) are satisfied.

Especially the latter fact is actually surprising and merits a remark. The composition property (7), when introduced in Section 2 seemed obviously correct. This was for the simple reason that the propagator (4) is a path integral, which by its very meaning should be sliceable into pieces if one integrates over all intermediate configurations. However, since the path integral is over "all" configurations (real functions) in spacetime, the configurations to be integrated over on the boundary between slices should be "all" configurations (real functions). This is not what we are doing. We are only integrating over the physical configurations as explained above. That the composition rule holds nevertheless is thus a non-trivial fact (in contrast to the spacelike case).

The normalization condition (10) on the vacuum yields now

$$
|C|^{-2}=\int \mathcal{D} \varphi \exp \left(-\frac{1}{2} \int \mathrm{d} t \mathrm{~d}^{2} \tilde{x} \varphi 2 \kappa_{1} \varphi\right) .
$$


One may argue that this yields the same $|C|$ as (20) by putting the two configurations spaces and their measures into correspondence. There is a novel aspect concerning the phase of $C$ here. Recall from (8) that the vacuum wave function on an oppositely oriented hyperplane must be given by the complex conjugate. However, we may use a spatial rotation to transform a timelike hyperplane into itself, but with opposite orientation. Thus, rotating the vacuum this way and requiring equality with the complex conjugate yields the condition that the vacuum wave function must be real. This implies $C$ to be real. ${ }^{3}$

\subsection{Timelike particle states}

After having convinced ourselves that the structures defined have all properties listed in Section 2 we move to consider particle states. Since a state is a wave function on physical configurations on the hyperplane, a basis of one-particle states may be characterized by the Fourier modes in this hyperplane. In the standard (spacelike) case these are labeled by 3 -momentum. In the present (timelike) case these are labeled by the (possibly negative) energy and the momentum in the $\tilde{x}$-directions. We will consider here merely the formal properties of particle states, postponing a discussion of their meaning to Section 6

Since we set the energy variable to be positive, $E>0$, we distinguish the actual sign of the energy by an index \pm , using a Fourier transform of the form

$$
\check{\varphi}^{ \pm}(E, \tilde{p}):=2 p_{1} \int \mathrm{d} t \mathrm{~d}^{2} \tilde{x} e^{ \pm \mathrm{i}(E t-\tilde{p} \tilde{x})} \varphi(t, \tilde{x}) .
$$

The one-particle state of energy $E$ (or $-E$ ) and 2-momentum $\tilde{p}$ is given by the wave function

$$
\psi_{E, \tilde{p}}^{ \pm}(\varphi)=\check{\varphi}^{ \pm}(E, \tilde{p}) \psi_{0}(\varphi) .
$$

Its eigenvalue under "spatial evolution" from $x_{1}$ to $x_{1}^{\prime}$ is given by $\exp \left(\mathrm{i} \Delta p_{1}\right)$, where $p_{1}$ is the positive square root $p_{1}=\sqrt{E^{2}-\tilde{p}^{2}-m^{2}}$. The inner product of one-particle states is given by the distribution

$$
\left\langle\psi_{E, \tilde{p}}^{a}, \psi_{E^{\prime}, \tilde{p}^{\prime}}^{a^{\prime}}\right\rangle=(2 \pi)^{3} 2 p_{1} \delta_{a, a^{\prime}} \delta\left(E-E^{\prime}\right) \delta^{2}\left(\tilde{p}-\tilde{p}^{\prime}\right) .
$$

The complex conjugation of the wave function associated with a change of orientation of the hyperplane simply changes the sign index,

$$
\overline{\check{\varphi}^{ \pm}(E, \tilde{p}) \psi_{0}(\varphi)}=\check{\varphi}^{\mp}(E, \tilde{p}) \psi_{0}(\varphi) .
$$

Multi-particle states are formed in analogy to the spacelike case, namely by starting with a monomial in (26) times the vacuum wave function and then projecting out the components in subspaces of lower particle number. For example,

\footnotetext{
${ }^{3}$ One might try to use a similar argument in the spacelike case using a time reflection transformation. However, this transformation is not connected to the identity of the Poincaré group and might thus not necessarily be expected to leave the vacuum invariant.
} 
the two-particle state takes the form

$$
\begin{aligned}
& \psi_{(E, \tilde{p}),\left(E^{\prime}, \tilde{p}^{\prime}\right)}^{a, a^{\prime}}(\varphi) \\
& \quad=\left(\check{\varphi}^{a}(E, \tilde{p}) \check{\varphi}^{a^{\prime}}\left(E^{\prime}, \tilde{p}^{\prime}\right)-(2 \pi)^{3} 2 p_{1} \delta_{a,-a^{\prime}} \delta\left(E-E^{\prime}\right) \delta^{2}\left(\tilde{p}-\tilde{p}^{\prime}\right)\right) \psi_{0}(\varphi) .
\end{aligned}
$$

Similarly to the spacelike case, we may identify state spaces associated to parallel hyperplanes by using a spatial translation symmetry. We may thus write amplitudes between such parallel hyperplanes. For example, the one-particle to one-particle amplitude is

$$
\begin{aligned}
\rho_{\left[x_{1}, x_{1}^{\prime}\right]}\left(\psi_{E, \tilde{p}}^{a} \otimes \psi_{E^{\prime}, \tilde{p}^{\prime}}^{a^{\prime}}\right) & \\
= & \int_{K_{x_{1}} \times K_{x_{1}^{\prime}}} \mathcal{D} \varphi \mathcal{D} \varphi^{\prime} \psi_{E, \tilde{p}}^{a}(\varphi) \psi_{E^{\prime}, \tilde{p}^{\prime}}^{a^{\prime}}\left(\varphi^{\prime}\right) Z_{\left[x_{1}, x_{1}^{\prime}\right]}\left(\varphi, \varphi^{\prime}\right) \\
& =e^{\mathrm{i} \Delta p_{1}}(2 \pi)^{3} 2 p_{1} \delta_{a,-a^{\prime}} \delta\left(E-E^{\prime}\right) \delta^{2}\left(\tilde{p}-\tilde{p}^{\prime}\right) .
\end{aligned}
$$

Note that no explicit complex conjugation appears here, since we have chosen the wave functions with respect to the orientations of the carrying hyperplanes as boundaries of the enclosed region.

As in the spacelike case we may rewrite the amplitude in a form analogous to (24), reminiscent of the bra-ket notation. However, this is no longer very useful. In contrast to the spacelike case, rotational symmetry prevents a consistent orientation of all timelike hypersurfaces from the outset. Thus, none of the two possible ways of of writing the amplitude (31) in the form (24), arising from the two possible orientations is preferred.

\subsection{General vacuum}

We now turn to arbitrary hyperplanes in Minkowski space. Such a hyperplane is either spacelike, timelike or null. In the first case we can obtain it by a Poincaré transformation from the equal-time hyperplane considered in Section [3] In the second case we can obtain it by a Poincaré transformation from the timelike hyperplane considered above. Since the theory is fully Poincaré covariant, state spaces, propagators etc. can all be obtained in a straightforward way by the induced transformations. We will not detail the results here, but limit ourselves to one object which indicates the consistency of the present approach in a surprising way. This is the vacuum wave function.

Consider an arbitrary hyperplane. Suppose the angle between time axis and the euclidean normal vector to the hyperplane is given by $\alpha$. Since the effect of translations and of spatial rotations is straightforward and uninteresting, we translate and rotate our coordinate system such that the hyperplane in question is spanned by coordinate directions $\left(s, x_{2}, x_{3}\right)$. Here $s$ is a Euclidean coordinate along the hyperplane such that $x_{1}=s \cos \alpha$ and $t=s \sin \alpha$.

It was shown in 1] using suitable Lorentz boosts, that both, in the spacelike as well as in the timelike case the vacuum wave function on the hyperplane may 
be written as

$$
\psi_{0}(\varphi)=C \exp \left(-\frac{1}{2} \int \mathrm{d} s \mathrm{~d}^{2} \tilde{x} \varphi(s, \tilde{x})(\tau \varphi)(s, \tilde{x})\right),
$$

where $\tau$ is an operator. What is more, the operator $\tau$ takes a simple form which covers both, the spacelike and the timelike case,

$$
\tau=\sqrt{-\partial_{s}^{2}+\cos 2 \alpha\left(-\sum_{i \geq 2} \partial_{i}^{2}+m^{2}\right)} .
$$

It is easy to see that for $\alpha=0$ we recover $\omega$ and for $\alpha=\pi / 2$ we recover $\kappa_{1}$. (This fixes the choice of sign encountered earlier.) Remarkably, however, the vacuum wave function depends smoothly on the angle $\alpha$, not only in the intervals $0 \leq \alpha<\pi / 4$ (spacelike) and $\pi / 4<\alpha \leq \pi / 2$ (timelike), but even at and near $\pi / 4$ (null). On the one hand this indicates that our separate treatments of the spacelike and timelike cases are indeed consistent with each other. On the other hand this suggests that even states on null hyperplanes may make sense.

\section{The hypercylinder}

In this section we consider hypersurfaces which are infinite hypercylinders (and hyperspheres) in the following sense. Consider a sphere of radius $R$ in space. Take the hypersurface formed by the extension of this sphere over all of time in Minkowski space. We will call this simply the hypercylinder of radius $R$. At the same time we consider regions of spacetime given by a solid hypercylinder $B_{R}$. Furthermore, we consider the solid hypercylinder (of radius $\hat{R}$ ) with a smaller solid hypercylinder (of radius $R$ ) cut out, denoting this region by $B_{[R, \hat{R}]}$.

\subsection{Coordinates, classical solutions etc.}

We use spherical coordinates in space, parametrized by angles $\theta \in[0, \pi[$ and $\phi \in[0,2 \pi[$ and the radius $r \in[0, \infty[$. Concretely, we use the coordinate transformations $x_{1}=r \sin \theta \cos \phi, x_{2}=r \sin \theta \sin \phi, x_{3}=r \cos \theta$. The Laplace operator $\triangle:=\sum_{i \geq 1} \partial_{i}^{2}$ takes the form $\triangle=\triangle_{r}+\triangle_{\Omega}$ with

$$
\triangle_{r}:=\frac{2}{r} \partial_{r}+\partial_{r}^{2} \quad \text { and } \quad \triangle_{\Omega}:=\frac{\cos \theta}{r^{2} \sin \theta} \partial_{\theta}+\frac{1}{r^{2} \sin \theta} \partial_{\theta}^{2}+\frac{1}{r^{2} \sin \theta} \partial_{\phi}^{2} .
$$

We can expand solutions of the equations of motions in terms of spherical harmonics via

$$
\phi(t, r, \Omega)=\int \mathrm{d} E \sum_{l=0}^{\infty} \sum_{m=-l}^{l} \alpha_{l, m}(E) e^{-\mathrm{i} E t} f_{l}(p r) Y_{l}^{m}(\Omega) .
$$

The integral over $E$ is constrained to $|E| \geq m$ and $p$ is the positive square root $p:=\sqrt{E^{2}-m^{2}} . \Omega$ is a collective notation for the angle coordinates $(\theta, \phi) . f_{l}$ 
denotes a spherical Bessel function of order $l$. We will consider spherical Bessel functions of the first kind, denoted $j_{l}$, and of the second kind, denoted $n_{l}$. The former describe globally defined solutions, while the latter describe solutions that are singular at the origin. We will also employ the spherical Bessel functions of the third kind (or Hankel functions) $h_{l}:=j_{l}+\mathrm{i} n_{l}$ and $\overline{h_{l}}:=j_{l}-\mathrm{i} n_{l}$.

$Y_{l}^{m}$ denotes the spherical harmonic defined through the associated Legendre function $P_{l}^{m}$ via

$$
Y_{l}^{m}(\theta, \phi):=\sqrt{\frac{(2 l+1)(l-m) !}{4 \pi(l+m) !}} P_{l}^{m}(\cos \theta) e^{\mathrm{i} m \phi} .
$$

Note that complex conjugation yields $\overline{Y_{l}^{m}}=Y_{l}^{-m}$. The spherical harmonics satisfy the orthogonality relation

$$
\int \mathrm{d} \Omega Y_{l}^{m} \overline{Y_{\hat{l}}^{\hat{m}}}=\delta_{l, \hat{l}} \delta_{m, \hat{m}}
$$

Here $\mathrm{d} \Omega:=\frac{1}{4 \pi} \mathrm{d} \phi \mathrm{d} \theta \sin \theta$. We also remark that

$$
\left(\triangle_{r} f_{l}\right)(p r)=\left(-p^{2}+\frac{l(l+1)}{r^{2}}\right) f_{l}(p r), \quad\left(\triangle_{\Omega} Y_{l}^{m}\right)(\Omega)=-\frac{l(l+1)}{r^{2}} Y_{l}^{m}(\Omega),
$$

where $f_{l}$ is any of the spherical Bessel functions.

As in the case of timelike hyperplanes the space of physical field configurations on a hypercylinder is the space of only those configurations that extend to a classical solution. From $|E| \geq m$ we can infer that the eigenvalues of the operator $-\partial_{0}^{2}$ on the physical configuration space must be larger or equal to $m^{2}$. Note that we parametrize all hypercylinders in the same way, irrespective of radius, namely via the solid angle $\Omega$ and the time $t$.

\subsection{Propagators}

We shall be interested in two types of propagation regions: the solid hypercylinder $B_{R}$ and the region $B_{[R, \hat{R}]}$ between two hypercylinders. In both cases we wish to evaluate the path integral (4) in the same way used in the cases of hyperplanes, namely using a classical solution matching the boundary data. This entails a seeming contradiction. Namely, if the field configuration on a single hypercylinder is in one-to-one correspondence to classical solutions then the combination of field configurations on two hypercylinders cannot be in oneto-one correspondence to classical solutions and vice versa. This apparent contradiction has the following resolution. We only require the classical solutions to be defined within the propagation region. This implies that for the solid hypercylinder $B_{R}$ we are restricted to the solutions (34) with spherical Bessel functions of the first kind. In contrast, the region $B_{[R, \hat{R}]}$ does not contain the time axis. Hence, in addition we may admit solutions arising from spherical Bessel functions of the second kind. We shall see that this somewhat heuristic procedure leads to a consistent picture. 
The propagator for the full hypercylinder is the easier one to work out. Note that the boundary version (13) of the action on a classical solution now takes the form

$$
S_{R}\left(\phi_{\mathrm{cl}}\right)=-\frac{1}{2} \int \mathrm{d} t \mathrm{~d} \Omega 4 \pi R^{2} \phi_{\mathrm{cl}}(t, R, \Omega)\left(\partial_{r} \phi_{\mathrm{cl}}\right)(t, R, \Omega) .
$$

Combining this with (34) using the spherical Bessel functions of the first kind yields the propagator

$$
Z_{R}(\varphi)=N_{R} \exp \left(-\frac{1}{2} \int \mathrm{d} t \mathrm{~d} \Omega 4 \pi \varphi(t, \Omega) \operatorname{ip} R^{2} \frac{j_{l}^{\prime}(p R)}{j_{l}(p R)} \varphi(t, \Omega)\right) .
$$

Here $j_{l}^{\prime}$ denotes the derivative of $j_{l}$. The expression

$$
p R^{2} \frac{j_{l}^{\prime}(p R)}{j_{l}(p R)}
$$

is to be understood as an operator defined through its eigenvalues on a mode expansion of the field configuration. To this end note that $p=\sqrt{E^{2}-m^{2}}$ can be extracted from the temporal plane wave mode expansion while $l$ can be extracted from the spherical harmonic mode expansion.

Consider now the region $B_{[R, \hat{R}]}$ between nested hypercylinders. The boundary form of the action is the difference of two terms of the form (36). To obtain a propagator we may start by splitting a classical solution into two components. For example, in terms of a regular and a singular component we have,

$$
\phi_{\mathrm{cl}}(t, r, \Omega)=j_{l}(p r) \varphi_{\text {reg. }}(t, \Omega)+n_{l}(p r) \varphi_{\text {sing. }}(t, \Omega) .
$$

Here $j_{l}(p r)$ and $n_{l}(p r)$ are understood as operators in the sense described above. The radial derivative yields

$$
\left(\partial_{r} \phi_{\mathrm{cl}}\right)(t, r, \Omega)=p j_{l}^{\prime}(p r) \varphi_{\text {reg. }}(t, \Omega)+p n_{l}^{\prime}(p r) \varphi_{\text {sing. }}(t, \Omega) .
$$

Using this and inverting the formal linear transformation

$$
\left(\begin{array}{l}
\varphi \\
\hat{\varphi}
\end{array}\right)=\left(\begin{array}{ll}
j_{l}(p R) & n_{l}(p R) \\
j_{l}(p \hat{R}) & n_{l}(p \hat{R})
\end{array}\right)\left(\begin{array}{l}
\varphi_{\text {reg. }} \\
\varphi_{\text {sing. }}
\end{array}\right)
$$

leads to the propagator

$$
Z_{[R, \hat{R}]}(\varphi, \hat{\varphi})=N_{[R, \hat{R}]} \exp \left(-\frac{1}{2} \int \mathrm{d} t \mathrm{~d} \Omega 4 \pi\left(\begin{array}{ll}
\varphi & \hat{\varphi}
\end{array}\right) W_{[R, \hat{R}]}\left(\begin{array}{l}
\varphi \\
\hat{\varphi}
\end{array}\right)\right)
$$

with

$$
W_{[R, \hat{R}]}:=\frac{\mathrm{i} p}{\delta_{l}(p R, p \hat{R})}\left(\begin{array}{cc}
R^{2} \sigma_{l}(p \hat{R}, p R) & -\frac{1}{p^{2}} \\
-\frac{1}{p^{2}} & \hat{R}^{2} \sigma_{l}(p R, p \hat{R})
\end{array}\right) .
$$

The functions $\delta_{l}$ and $\sigma_{l}$ are to be understood as operators and have the following definitions:

$$
\begin{aligned}
\delta_{l}(z, \hat{z}) & =j_{l}(z) n_{l}(\hat{z})-n_{l}(z) j_{l}(\hat{z})=\frac{\mathrm{i}}{2}\left(h_{l}(z) \overline{h_{l}}(\hat{z})-\overline{h_{l}}(z) h_{l}(\hat{z})\right) \\
\sigma_{l}(z, \hat{z}) & =j_{l}(z) n_{l}^{\prime}(\hat{z})-n_{l}(z) j_{l}^{\prime}(\hat{z})=\frac{\mathrm{i}}{2}\left(h_{l}(z){\overline{h_{l}}}^{\prime}(\hat{z})-\overline{h_{l}}(z) h_{l}^{\prime}(\hat{z})\right) .
\end{aligned}
$$




\section{$5.3 \quad$ Vacuum}

We now turn to the question of the vacuum state on the hypercylinder. Again we make a Gaussian ansatz of the form (18). The precise form is now

$$
\psi_{R, 0}^{I}(\varphi)=C_{R} \exp \left(-\frac{1}{2} \int \mathrm{d} t \mathrm{~d} \Omega 4 \pi \varphi(t, \Omega)\left(B_{R}^{I} \varphi\right)(t, \Omega)\right) .
$$

Here $B_{R}^{I}$ denotes a family of operators indexed by the radius $R$. Recall that a state with given physical meaning changes depending on the orientation of the carrying hypersurface with respect to the propagation region. Furthermore, in contrast to the case of hyperplanes, a hypercylinder with given orientation is not related to the hypercylinder with opposite orientation by any symmetry. Thus, we must a priori expect the vacuum wave function to be different for the two orientations. We have indicated this above by the superscript $I$, with $\psi_{R, 0}^{I}$ being the vacuum on the inner side of the hypercylinder. Correspondingly, we denote by $\psi_{R, 0}^{O}$ the vacuum on the outside and by $B_{R}^{O}$ the associated family of operators. Of course we expect the two vacua to satisfy (8), i.e., to be related by complex conjugation, $\overline{B_{R}^{O}}=B_{R}^{I}$. We also suppose that we can choose $C_{R}$ to be real.

An obvious condition to be satisfied by the operators $B_{R}^{I}$ (or $B_{R}^{O}$ ) is that they must be related to each other, for different radii, by propagation via (38). This condition is weaker than a full invariance condition, which was essentially sufficient to determine the vacuum in the case of hyperplanes. We might thus expect to require additional conditions to determine the vacuum uniquely. In any case, the propagation condition leads to the equation

$$
\left(z^{2} \sigma_{l}(\hat{z}, z)-\mathrm{i} p \delta_{l}(z, \hat{z}) B_{R}^{I}\right)\left(\hat{z}^{2} \sigma_{l}(z, \hat{z})+\mathrm{i} p \delta_{l}(z, \hat{z}) B_{\hat{R}}^{I}\right)=1,
$$

with $z:=p R$ and $\hat{z}:=p \hat{R}$.

For the general boundary formulation to be consistent over various topologies and geometries of hypersurfaces we would like the vacuum to be determined "locally" in a suitable sense. That is, the dependence of the vacuum wave function on the field configuration on a small piece of a hypersurface should be independent of the global topological or geometrical nature of the hypersurface. Furthermore, we would like the vacuum functional to "change smoothly" under "smooth changes" of the hypersurface. In terms of the ansatz (18) we desire these properties of the operator $A$. In the concrete case at hand we may use this to demand that the operator $B_{R}^{I}$ for large radii approximates the operator $\kappa_{1}$ (and its appropriately rotated versions) which was found to describe the vacuum on timelike hyperplanes.

Concretely, consider a small region near the positive $x_{1}$ axis at large fixed radius $R$, i.e., in spherical coordinates $\phi \approx 0$ and $\theta \approx \pi / 2$ at $r=R$. There, $\partial_{2} \approx \frac{1}{R} \partial_{\phi}$ and $\partial_{3} \approx \frac{1}{R} \partial_{\theta}$. Thus, $\partial_{2}^{2}+\partial_{3}^{2} \approx \frac{1}{R^{2}}\left(\partial_{\phi}^{2}+\partial_{\theta}^{2}\right)=\triangle_{\Omega}$. We thus demand

$$
B_{R}^{I} \approx R^{2} \sqrt{-\partial_{0}^{2}+\triangle_{\Omega}-m^{2}}
$$


for large $R$ in a suitable sense. Note that in this form the condition is rotationally invariant, independent of our initial consideration of a specific spatial direction (namely the $x_{1}$-axis). The factor $R^{2}$ comes from the differently scaled integration measures on the hyperplane versus the hypercylinder. In terms of eigenvalues on spherical harmonics and plane temporal waves the expression (40) takes the form

$$
B_{R}^{I} \approx R^{2} \sqrt{p^{2}-\frac{l(l+1)}{R^{2}}} \underset{R \rightarrow \infty}{\longrightarrow} p R^{2} .
$$

The indicated limit is understood with respect to fixed eigenvalues $p$ and $l$.

Our strategy is thus to take the condition (39), solve for $B_{R}^{I}$, insert $B_{\hat{R}}^{I}=p \hat{R}^{2}$ and evaluate the limit $\hat{R} \rightarrow \infty$. Indeed, the limit exists and the solution is

$$
B_{R}^{I}=\frac{1+\mathrm{i} z^{2}\left(j_{l}(z) j_{l}^{\prime}(z)+n_{l}(z) n_{l}^{\prime}(z)\right)}{p\left(j_{l}^{2}(z)+n_{l}^{2}(z)\right)}=\frac{1+\frac{\mathrm{i} z^{2}}{2}\left(h_{l}(z){\overline{h_{l}}}^{\prime}(z)+\overline{h_{l}}(z) h_{l}^{\prime}(z)\right)}{p h_{l}(z) \overline{h_{l}}(z)} .
$$

Reinserting this for $B_{R}^{I}$ and $B_{\hat{R}}^{I}$ into (39) confirms that we have found an actual solution of this equation. We remark also that, as is easy to see, there is another solution to (39) given by $-\overline{B_{R}^{I}}$ which asymptotically approximates $-\kappa_{1}$, thus recovering the ambiguity encountered earlier.

Note that $B_{R}^{I}$ turns out to be a rational function of $z$, without singularities for positive $z$. Moreover, the operator $z^{2}\left(j_{l}^{2}(z)+n_{l}^{2}(z)\right)$ can be expressed in terms of $p^{2}$ and $\triangle_{\Omega}$ via a sum as follows,

$$
z^{2}\left(j_{l}^{2}(z)+n_{l}^{2}(z)\right)=\sum_{k=0}^{\infty} \prod_{j=0}^{k-1} \frac{2 j+1}{2 j+2} \frac{1}{p^{2}}\left(-\triangle_{\Omega}-\frac{j(j+1)}{R^{2}}\right) .
$$

This can be derived using the eigenvalues [35] and suitable facts about spherical Bessel functions, see e.g. [7.

Turning to the outside version of the vacuum, we observe that the relevant propagator (from $\hat{R}$ to $R$ ) is the same as (38) except for the overall sign of the exponent. Since the exponent is purely imaginary this corresponds to a complex conjugation and the resulting relation between $B_{R}^{O}$ and $B_{\hat{R}}^{O}$ is simply the complex conjugate of (39). Consequently, $B_{R}^{O}:=\overline{B_{R}^{I}}$ solves the condition. Furthermore, it obviously has the same asymptotic limit as $B_{R}^{I}$ and is thus the required outside vacuum. Hence, the vacuum satisfies the conjugation condition (8) as expected. One may speculate that the imaginary parts of $B_{R}^{I}$ and $B_{R}^{O}$ are related to the curvature of the hypersurface.

The normalization condition (10) for the vacuum yields

$$
\left|C_{R}\right|^{-2}=\int \mathcal{D} \varphi \exp \left(-\frac{1}{2} \int \mathrm{d} t \mathrm{~d} \Omega 4 \pi \varphi(t, \Omega)\left(\frac{2}{p h_{l}(p R) \overline{h_{l}}(p R)} \varphi\right)(t, \Omega)\right) .
$$

In turn we can use this to determine the normalization of the propagators. By condition (11) the contraction of the outside vacuum with the full hypercylinder 
propagator (37) should give one. This implies

$$
N_{R}^{-1}=\bar{C}_{R}^{-1} \int \mathcal{D} \varphi \exp \left(-\frac{1}{2} \int \mathrm{d} t \mathrm{~d} \Omega 4 \pi \varphi(t, \Omega)\left(\frac{1}{p j_{l}(p R) h_{l}(p R)} \varphi\right)(t, \Omega)\right) .
$$

For the normalization of the propagator for the nested hypercylinders we obtain

$$
\begin{aligned}
N_{[R, \hat{R}]}^{-1} & =C_{R} C_{\hat{R}}^{-1} \\
& \int \mathcal{D} \varphi \exp \left(-\frac{1}{2} \int \mathrm{d} t \mathrm{~d} \Omega 4 \pi \varphi(t, \Omega)\left(\frac{\mathrm{i} \overline{h_{l}}(p \hat{R})}{p \overline{h_{l}}(p R) \delta_{l}(p R, p \hat{R})} \varphi\right)(t, \Omega)\right) .
\end{aligned}
$$

One can now check that the unitarity condition (6) is satisfied for both types of propagators. Furthermore, the composition property (7) is satisfied both for composing (38) with itself as well as for composing (38) with (37). As in the case of timelike hyperplanes, the restriction of configuration spaces makes the validity of the composition rule a non-trivial result.

\subsection{Particle states}

We now turn to particle states. As in the case of timelike hyperplanes, we present here a purely technical discussion, postponing the interpretational questions to Section 6

We start with the one-particle state. We consider first an "outside" state. The state may be characterized in terms of the mode expansion, i.e., through its energy $E$ and angular momentum "quantum numbers" $l$ and $m$. Set

$$
\check{\varphi}_{R, l, m}^{ \pm}(E)=\int \mathrm{d} t \mathrm{~d} \Omega 4 \pi \frac{\sqrt{2}}{\sqrt{p}\left|h_{l}(p R)\right|} e^{ \pm \mathrm{i} E t} Y_{l}^{\mp m}(\Omega) \varphi(t, \Omega) .
$$

As in (26) we set $E>0$ and encode the sign of the energy through a separate index. With this a one-particle state reads

$$
\psi_{R, E, l, m}^{O, \pm}(\varphi)=\check{\varphi}_{R, l, m}^{ \pm}(E) \psi_{R, 0}^{O}(\varphi) .
$$

The inner product is given by

$$
\left\langle\psi_{R, E, l, m}^{O, a}, \psi_{R, E^{\prime}, l^{\prime}, m^{\prime}}^{O, a^{\prime}}\right\rangle_{R}^{O}=8 \pi^{2} \delta\left(E-E^{\prime}\right) \delta_{l, l^{\prime}} \delta_{m, m^{\prime}} \delta_{a, a^{\prime}} .
$$

The corresponding "inside" state is the complex conjugate, i.e.,

$$
\psi_{R, E, l, m}^{I, \pm}(\varphi)=\overline{\psi_{R, E, l, m}^{O, \pm}(\varphi)}=\overline{\check{\varphi}_{R, l, m}^{ \pm}(E) \psi_{R, 0}^{O}(\varphi)}=\check{\varphi}_{R, l, m}^{\mp}(E) \psi_{R, 0}^{I}(\varphi) .
$$

The inner product is the same as (42), with index $O$ replaced by index $I$.

An "outside" two-particle state is given by

$$
\begin{aligned}
& \psi_{R,(E, l, m),\left(E^{\prime}, l^{\prime}, m^{\prime}\right)}^{O, a, a^{\prime}}(\varphi)= \\
& \quad\left(\check{\varphi}_{R, l, m}^{a}(E) \check{\varphi}_{R, l^{\prime}, m^{\prime}}^{a^{\prime}}\left(E^{\prime}\right)-8 \pi^{2} \delta\left(E-E^{\prime}\right) \delta_{l, l^{\prime}} \delta_{m, m^{\prime}} \delta_{a,-a^{\prime}}\right) \psi_{R, 0}^{O}(\varphi) .
\end{aligned}
$$


Multi-particle states are obtained in analogy to the procedure in the case of hyperplanes, i.e., by starting with the suitable monomial times the vacuum as the wave function and projecting out particle states of lower particle number.

Amplitudes may now be associated with two different types of regions. On the one hand, we have "transition" amplitudes between hypercylinders of different radii via the propagator (38). This is somewhat analogous to the propagation between parallel hyperplanes although with the difference that the two hypersurfaces in question are not isometric. On the other hand we have the conceptually novel possibility of considering amplitudes for a single hypercylinder via its solid propagator (37). This amplitude cannot be written as a "transition" amplitude between hypersurfaces in the sense of (5).

The boundary of the region $B_{[R, \hat{R}]}$ consists of two hypercylinders. As boundaries they are oriented. Concretely, the smaller hypercylinder of radius $R$ is oriented "inside" and the larger hypercylinder of radius $\hat{R}$ is oriented "outside". Given an "inside" one-particle state at radius $R$ and an "outside" one-particle state at radius $\hat{R}$ yields

$$
\rho_{[R, \hat{R}]}\left(\psi_{R, E, l, m}^{I, a}, \psi_{\hat{R}, E^{\prime}, l^{\prime}, m^{\prime}}^{O, a^{\prime}}\right)=\alpha_{[R, \hat{R}], p, l} 8 \pi^{2} \delta\left(E-E^{\prime}\right) \delta_{l, l^{\prime}} \delta_{m, m^{\prime}} \delta_{a, a^{\prime}} .
$$

Here,

$$
\alpha_{[R, \hat{R}], p, l}:=\frac{h_{l}(p \hat{R})}{h_{l}(p R)} \frac{\left|h_{l}(p R)\right|}{\left|h_{l}(p \hat{R})\right|}=\frac{\overline{h_{l}}(p R)}{\overline{h_{l}}(p \hat{R})} \frac{\left|h_{l}(p \hat{R})\right|}{\left|h_{l}(p R)\right|} .
$$

As an example of an amplitude for the solid hypercylinder using (37) we evaluate the two-particle state shown above,

$$
\rho_{R}\left(\psi_{R,(E, l, m),\left(E^{\prime}, l^{\prime}, m^{\prime}\right)}^{O, a, a^{\prime}}\right)=\frac{h_{l}(p R)}{\overline{h_{l}}(p R)} 8 \pi^{2} \delta\left(E-E^{\prime}\right) \delta_{l, l^{\prime}} \delta_{m, m^{\prime}} \delta_{a,-a^{\prime}}
$$

Note that this type of amplitude is defined exclusively for "outside" states.

\section{Interpretation}

So far we have dealt with the formal side of the general boundary formulation showing that a consistent picture of states, vacua, amplitudes etc. emerges for the hypersurfaces and regions considered. In terms of the companion paper [2], all the core axioms as well as the vacuum axioms are satisfied. We now turn to the physical interpretation of those structures.

\subsection{Particles on timelike hypersurfaces}

An initial discussion of particle states on timelike hypersurfaces was already given in [1] (in the hyperplane case). We review parts of this discussion here and add the novel aspects arising from the hypercylinder case.

A crucial difference between states on spacelike and timelike hypersurfaces arises as follows. In the spacelike case causality implies that a state is purely an 
incoming state or an outgoing state depending on whether it forms the beginning or the end of a time-evolution process. This distinction is encoded in the standard formulation by whether the state is a ket-state (in-state) or a bra-state (out-state). We have linked this distinction in Section 3 to the orientation (past or future) of the carrying hypersurface. In contrast, in the timelike case, a state on a given oriented hypersurface is neither necessarily an incoming state nor an outgoing one. Rather, each particle within the state may be independently incoming or outgoing. This choice is precisely given by the sign of the energy in (26) and (41), encoded by the index \pm . We set the negative sign to represent in-particles and the positive sign to represent out-particles. (This makes momenta on spacelike and timelike hyperplanes mutually consistent [1.)

In the case of particles on hypercylinders the parametrization of particle states we have chosen makes immediate sense, thinking in terms of classical waves expanded spherically. In particular, (away from the center) it is natural to think in terms of incoming or outgoing waves. In the hyperplane case we are more accustomed to think in terms of a plane wave expansion parametrized by 3 -momenta $p$. However, the parametrization in terms of energy $E$, incoming versus outgoing \pm and 2-momentum $\tilde{p}$ is easily related. Indeed, the equation $E^{2}=p_{1}^{2}+\tilde{p}^{2}+m^{2}$ determines the missing momentum component $p_{1}$ already up to a sign. This sign is now determined indirectly by \pm . Namely, the momentum of an in-particle has to point into the propagation region while that of an outparticle has to point out of the propagation region. Hence, for an in-particle on an oriented hyperplane $p_{1}$ is directed to the opposite side of the hyperplane, while for an out-particle it is directed to the same side.

Note also that the relation to orientation change via complex conjugation (2) is consistent. Namely, an in-particle considered on a hyperplane with opposite orientation must become an out-particle since the propagation region is now on the other side. Correspondingly for out-particles. As we have seen in (29) and (43), this is precisely what happens.

The amplitudes we have calculated are also consistent with the in/out interpretation of particle states we have given. Recall that in the non-interacting theory we are considering, amplitudes simply express all the possibilities in which particles on the boundary could be identical. In the hyperplane case, this means that an in-particle on one hyperplane can only pair with an out-particle on the other one and vice versa. This is exactly what we see in (31). The situation is similar for two nested hypercylinders, namely an in-particle on one hypercylinder can only pair with an out-particle on the other one and vice versa, see (44). A different situation arises for the solid hypercylinder. Classically, an incoming spherical wave produces an outgoing one and vice versa. Indeed, we see in this case that in-particles only pair with out-particles and vice versa, see (45).

\subsection{Probability interpretation}

In the standard formulation the modulus square of a transition amplitude from a state $\psi$ to a state $\eta$ is the probability of observing the final state $\eta$ (rather 
than its orthogonal complement) given that the state $\psi$ was prepared initially. Obviously, this interpretation is not applicable to generic situations arising in the proposed formulation, where an amplitude may be evaluated on a single state space.

In cases where we have a region bounded by two disjoint hyperplanes we can still use the standard interpretation with minimal change. For example, consider the case of two parallel timelike hyperplanes. The modulus square of an amplitude of the type (31) provides the probability of "observing" a state on one hyperplane given that another one was "prepared" on the other hyperplane. (In the case at hand one state is $\psi_{E, \tilde{p}}^{a}$ and the other one is $\psi_{E^{\prime}, \tilde{p}^{\prime}}^{a^{\prime}}$ ) Nevertheless, a crucial difference to the standard formulation is that the definite temporal character of the procedure is lost. That is, "preparation" no longer necessarily precedes "observation". Rather, we are dealing with a more general conditional probability. The amplitudes for regions between nested hypercylinders might be interpreted similarly, namely, as transition amplitudes from one hypercylinder to the other one. Correspondingly the associated probability may be interpreted as that of one state being measured on one hypercylinder conditional on another state being present on the other hypercylinder.

A probability interpretation for the general case is proposed in the companion paper 2. We briefly recall it in its general form. Let $M$ be a region with boundary $\Sigma$, the associated state space being $\mathcal{H}_{\Sigma}$. We denote the amplitude by $\rho_{M}: \mathcal{H}_{\Sigma} \rightarrow \mathbb{C}$. We specify part of a measurement process through a closed subspace $\mathcal{S} \subset \mathcal{H}_{\Sigma}$. This may be thought of as representing certain knowledge about the process (compare to "preparation"). Furthermore, we specify a second closed subspace $\mathcal{A} \subseteq \mathcal{S}$. This may be thought of as representing a question posed in the process, namely, whether the state corresponding to the measurement is in the subspace $\mathcal{A}$ (rather than in its orthogonal complement) given that it is in $\mathcal{S}$ (compare to "observation"). Let $\left\{\xi_{i}\right\}_{i \in I}$ be an orthonormal basis of $\mathcal{S}$, which reduces to an orthonormal basis of $\mathcal{A}$ given by $\left\{\xi_{i}\right\}_{i \in J \subseteq I}$. The probability $P(\mathcal{A} \mid \mathcal{S})$ associated with the process is given by the quotient,

$$
P(\mathcal{A} \mid \mathcal{S})=\frac{\sum_{i \in J}\left|\rho_{M}\left(\xi_{i}\right)\right|^{2}}{\sum_{i \in I}\left|\rho_{M}\left(\xi_{i}\right)\right|^{2}} .
$$

It is shown in 2] that this interpretation can be reduced to the standard one in the standard circumstances (time-evolution between spacelike hyperplanes). Furthermore, it covers some less standard conditional probabilities that can be inferred from the standard ones. Here, however, we shall be interested in a genuinely non-standard application where the boundary of the region associated with the measurement is connected.

Consider a hypercylinder of radius $R$. Call the associated outside state space $\mathcal{H}$. Given a function $f_{l, m}(E)$ satisfying

$$
8 \pi^{2} \int \mathrm{d} E \sum_{l, m}\left|f_{l, m}(E)\right|^{2}=1,
$$


the one-particle states defined by the wave functions

$$
\psi_{f}^{O, \pm}(\varphi):=\int \mathrm{d} E \sum_{l, m} f_{l, m}(E) \psi_{R, E, l, m}^{O, \pm}(\varphi)
$$

are normalized due to (42). Similarly, taking another function $f_{l, m}^{\prime}(E)$ satisfying (47), the two-particle states given by

$$
\psi_{f, f^{\prime}}^{O, a, a^{\prime}}(\varphi):=\int \mathrm{d} E \mathrm{~d} E^{\prime} \sum_{l, m, l^{\prime}, m^{\prime}} f_{l, m}(E) f_{l^{\prime}, m^{\prime}}^{\prime}\left(E^{\prime}\right) \psi_{R,(E, l, m),\left(E^{\prime}, l^{\prime}, m^{\prime}\right)}^{O, a, a^{\prime}}(\varphi)
$$

are normalized.

Now remember that $\mathcal{H}$ is a Fock space and may be decomposed into a direct sum of components $\mathcal{H}_{n}$ with given particle number $n$, i.e., $\mathcal{H}=\bigoplus_{n=0}^{\infty} \mathcal{H}_{n}$. Now define a closed subspace $\mathcal{S}_{f} \subset \mathcal{H}$ as follows,

$$
\mathcal{S}_{f}:=\left\{\eta \in \mathcal{H}_{2} \mid \exists \lambda \in \mathbb{C}, f^{\prime}, a^{\prime}: \eta=\lambda \psi_{f, f^{\prime}}^{O,-, a^{\prime}}\right\}
$$

Here $f^{\prime}$ is supposed to be a function satisfying (47). In words this means the following: $\mathcal{S}_{f}$ is the subspace of the space of two-particle states where one particle is an in-particle that can be described by the function $f$ in the sense given above. For example, $f$ might be peaked around a particular energy and particular angular momentum quantum numbers, thus describing a particle wave packet with approximately these properties.

Let the subset $\mathcal{A}_{f, g} \subset \mathcal{S}_{f}$ be spanned by the single state $\psi_{f, g}^{O,-,+}$ for a function $g$ satisfying (47). As we will explain later, it turns out that the denominator of (46) is equal to one in the present case. This implies,

$$
P\left(\mathcal{A}_{f, g} \mid \mathcal{S}_{f}\right)=\left|\rho_{R}\left(\psi_{f, g}^{O,-,+}\right)\right|^{2}=\left|8 \pi^{2} \int \mathrm{d} E \sum_{l, m} \frac{h_{l}(p R)}{\overline{h_{l}}(p R)} f_{l, m}(E) g_{l, m}(E)\right|^{2}
$$

Note that due to the normalization (47) and the fact that $h_{l}(p R) / \overline{h_{l}}(p R)$ has modulus one this quantity is less or equal than one as required.

Physically, $P\left(\mathcal{A}_{f, g} \mid \mathcal{S}_{f}\right)$ is the probability that we "observe" an outgoing particle characterized by a wave packet determined by $g$, given that an incoming particle with wave packet determined by $f$ was "prepared". Note that we could also reverse the role of the incoming and the outgoing particle. However, the meaning of "preparing" and "observing" would be less intuitive then. It is clear that we can extend this example to multi-particle states. That is, we can define a subspace $\mathcal{S}$ such that the total number of particles is fixed and some of them have determined wave packets. We can then "test" via $\mathcal{A}$ for specific wave packets for the remaining particles.

Considering certain outgoing particles conditional on certain incoming ones is of course what one usually does in perturbative quantum field theory. The difference is that in the standard formulation these particles live in different state 
spaces. However, we can artificially produce a similar situation here. It turns out that the Fock space $\mathcal{H}$ may be decomposed into a product of a Fock space of incoming particles $\mathcal{H}^{-}$and one of outgoing particles $\mathcal{H}^{+}$, i.e., $\mathcal{H}=\mathcal{H}^{-} \otimes \mathcal{H}^{+}$. Indeed, we may construct $\mathcal{H}^{+}$and $\mathcal{H}^{-}$in terms of the respective subspaces of $\mathcal{H}$. Note that these inherit inner products in this way and are isomorphic. Also, since incoming and outgoing particles are mutually orthogonal, the inner product of $\mathcal{H}$ is identical to that reconstructed from those of $\mathcal{H}^{-}$and $\mathcal{H}^{+}$.

What is more, defining $\overline{\mathcal{H}}^{+}$to be the dual Hilbert space of $\mathcal{H}^{+}$it turns out that the map $\tilde{\rho}_{R}: \mathcal{H}^{-} \rightarrow \overline{\mathcal{H}}^{+}$induced by the amplitude is not only well defined, but preserves the inner product. Thus, the decomposition $\mathcal{H}=\mathcal{H}^{-} \otimes \mathcal{H}^{+}$ behaves exactly as if it was induced by a decomposition of the carrying hypersurface. (This is also the reason why the denominator of the probability expression (48) is equal to one.) We recover a description which shows resemblance to the standard formulation. However, the map $\tilde{\rho}_{R}$ is of course not simply a "time-evolution".

\section{Conclusions and Outlook}

We hope to have presented in this work a compelling example of a general boundary quantum field theory in the shape of the Klein-Gordon theory. The regions and hypersurfaces considered are still much less general than what one would like to allow (see also the discussion in 2] on this topic). However, the hypercylinder case in particular exhibits many of the novel and non-standard features of the general boundary formulation. This also included the first concrete application of the generalized probability interpretation proposed in [2] in a context beyond the reach of standard quantum mechanics.

Note that the Fock space structure of the state space was instrumental in the probability interpretation of the solid hypercylinder example. It allowed to construct subsets of the state space with a clear physical meaning is a simple way. It might be expected that this will be a much more difficult problem in genuinely non-perturbative theories, where no convenient grading of the state space is available. For example, in the case of gravity, it is a priori highly unclear which properties of the (quantum) geometry of a hypersurface we may set fixed and for which sub-properties we may then meaningfully "ask" in a measurement process.

The Klein-Gordon theory is obviously only a starting point and more complicated quantum field theories should be considered. However, we expect that for free theories this should be relatively straightforward. See, e.g., the discussion of spinor and gauge fields in the Schrödinger representation in [6]. For interacting theories, we expect that the usual perturbative approach can be carried over. In particular, it should be possible to derive the S-matrix through an infinite radius limit of the solid hypercylinder amplitude. Indeed, one might argue that this would be conceptually more satisfactory than the usual derivation from equal-time hyperplanes at large negative and positive times. Namely, considering the interaction to be negligible at large distances in space (from the 
experiment) appears more natural than considering it negligible at very early or late times. In particular, this would be compatible with genuinely static processes. Note that such a derivation would make the crossing symmetry of the S-matrix manifest, since incoming and outgoing particles are part of a single state. Indeed, precisely for this reason, crossing symmetry was taken in 3 as a strong indication for the validity of the general boundary formulation.

\section{References}

[1] R. Oeckl, States on timelike hypersurfaces in quantum field theory, Phys. Lett. B 622 (2005), 172-177, hep-th/0505267.

[2] R. Oeckl, General boundary quantum field theory: Foundations and probability interpretation, Preprint hep-th/0509122.

[3] R. Oeckl, Schrödinger's cat and the clock: Lessons for quantum gravity, Class. Quantum Grav. 20 (2003), 5371-5380, gr-qc/0306007.

[4] R. Oeckl, A "general boundary" formulation for quantum mechanics and quantum gravity, Phys. Lett. B 575 (2003), 318-324, hep-th/0306025.

[5] R. P. Feynman, Space-time approach to non-relativistic quantum mechanics, Rev. Mod. Phys. 20 (1948), 367-387.

[6] R. Jackiw, Analysis of infinite-dimensional manifolds-Schrödinger representation for quantized fields, Field theory and particle physics (Campos do Jordão, 1989), World Scientific, River Edge, 1990, pp. 78-143.

[7] M. Abramowitz and I. A. Stegun, Handbook of mathematical functions, U.S. Government Printing Office, Washington, 1964. 